\title{
A comparison of genome cohort participants' genetic knowledge and preferences to receive genetic results before and after a genetics workshop
}

\author{
Kayono Yamamoto ${ }^{1,2} \cdot$ Atsushi Shimizu $^{3} \cdot$ Fumie Aizawa $^{4} \cdot$ Hiroshi Kawame $^{5} \cdot$ Tomoharu Tokutomi $^{1,2}$. \\ Akimune Fukushima ${ }^{1,2}$
}

Received: 9 March 2018 / Revised: 27 May 2018 / Accepted: 8 July 2018 / Published online: 5 September 2018

(c) The Author(s) 2018. This article is published with open access

\begin{abstract}
Several biobanks have begun returning genetic results to individuals, making the development of public genetic literacy an urgent task for their effective use. No research exists regarding the effects of genetic education on biobank participants, so we conducted genetics workshops with specialists, and surveyed differences in the participants' $(n=112)$ preferences to receive their own genetic information by disease categories and their genetic knowledge using questionnaires before and after the workshops. Almost $90 \%$ of our participants were over 60 years old, which was similar to our previous preference research. The preference to receive five of the six categories of genetic information (lifestyle diseases, pharmacogenetics, adult-onset non-clinically actionable diseases, non-clinically actionable multifactorial diseases, and all genetic information) was slightly but significantly decreased after the genetics workshop. More participants preferred to receive genetic results regarding lifestyle diseases, pharmacogenetics, and adult-onset clinically actionable diseases after the workshop, while less participants preferred to receive information regarding adult-onset non-clinically actionable diseases, non-clinically actionable multifactorial diseases, and all genetic information. Total genetic knowledge scores significantly increased after the workshop (before: 11.89 , after: $13.30, p<0.001$ ). Our findings suggest that genetics workshops are useful to improve the genetic literacy of genome cohort participants.
\end{abstract}

Electronic supplementary material The online version of this article (https://doi.org/10.1038/s10038-018-0494-z) contains supplementary material, which is available to authorized users.

Akimune Fukushima

kyamamot@iwate-med.ac.jp

1 Division of Innovation and Education, Iwate Tohoku Medical Megabank Organization, Disaster Reconstruction Center, Iwate Medical University, Iwate, Japan

2 Department of Clinical Genetics, School of Medicine, Iwate Medical University, Iwate, Japan

3 Division of Biomedical Information Analysis, Iwate Tohoku Medical Megabank Organization, Disaster Reconstruction Center, Iwate Medical University, Iwate, Japan

4 Center for Liberal Arts and Sciences, Iwate Medical University, Iwate, Japan

5 Department of Education and Training, Tohoku Medical Megabank Organization, Tohoku University, Sendai, Japan

\section{Introduction}

The technological development of next-generation sequencing and bioinformatics has resulted in whole-genome/ exome sequencing being conducted in clinical settings [1]. Large-scale genomic screens have attempted to biobank DNA samples and identify various gene mutations that cause clinically actionable genetic diseases [2]. Several biobanks have begun returning results to individuals. The Coriell Personalized Medicine Collaborative, which is based on both disease-specific and healthy cohort studies, has begun reporting the relative risk for common complex diseases to the participants [3]. A few population-based biobank studies on single-gene diseases have also provided subjects with their genetic results. Haukkala et al. reported on the returning of genetic results regarding heritable long QT syndrome in a Finnish population-based cohort study in which $65 \%(n=17)$ of subjects positive for long QT syndrome, as detected by genotyping, participated in a followup study. Many participants were surprised by the positive result, but deemed the information useful for their health 
[4]. The Tohoku Medical Megabank Project, a populationbased biobank in Japan [5], has begun returning individual genetic information regarding familial hypercholesterolemia. We have previously studied the population-based biobank participants' preferences to receive genetic test results, revealing that nearly $90 \%$ of participants enrolled in the Tohoku Medical Megabank Project cohort study expressed a preference to receive their results [6]. However, participant preferences based on disease categories have not been systematically studied.

During the last decade, human genetics technology has dramatically progressed and genetics-centered health-care is becoming a reality. Therefore, developing public genetic literacy is urgently required to help people understand their genetic information and use it rationally and effectively [7]. Appropriate science education programs have been developed in schools $[8,9]$ and several genetic education programs are being offered in schools and communities [10]. Intervention studies have also examined the effects of genetic lectures conducted by primary care providers [11]. However, there is no research regarding the effects of genetic education on biobank participants. Therefore, we surveyed the differences among participant preferences to receive their own genetic information by disease categories, and examined the effects of participation in a basic genetics workshop run by genetic specialists who usually offer genetic counseling in clinical situations on the participants' genetic knowledge, and preferences to receive genetic results.

\section{Materials and methods}

\section{Study population}

The study population was recruited from participants who attended health checks at Iwate Tohoku Medical Megabank satellites (October 2014 to February 2015). We distributed a research participant recruitment leaflet to health check participants, and introduced the details of our research to people who visited our recruitment booth. We conducted an initial face-to-face questionnaire (the before workshop questionnaire) after obtaining informed consent. At a later date, we conducted a workshop providing genetic knowledge to respondents of the first questionnaire, and then repeated the face-to-face questionnaire with the workshop participants (the after-workshop questionnaire).

\section{Questionnaire development and administration}

In this study, we analyzed participants' responses regarding preferences to receive their genetic test results. We examined differences in the participants' preferences to receive their own genetic information by disease category, the reasons for the participants' preferences, and the participants' preferred method of receiving their genetic information. We also investigated the participants' genetic knowledge and demographic data.

We developed the questions regarding preferences to receive genetic test results using a literature review, expert consultations, and a pilot study. The detailed process has been previously described [6]. The participants' preferences regarding the receipt of their genetic information were examined for the following types of diseases: lifestyle diseases (e.g., hypertension, diabetes), pharmacogenetics (e.g., responses to cold remedies, anticancer agents), adult-onset clinically actionable diseases (e.g., hereditary breast and ovarian cancer, hereditary colorectal cancer), adult-onset non-clinically actionable diseases, non-clinically actionable diseases in which the appearance of symptoms is not solely determined by genetics (e.g., Alzheimer's disease), and all genetic information, regardless of its relation to disease. We did not give an example regarding adult-onset non-clinically actionable diseases because describing specific diseases to be non-clinically actionable in the questionnaire can be offensive to participants affected with the disease. We used a 4-point Likert-type scale, where $1=$ "I want to know", $2=$ "If I must answer, then I want to know", 3 = "If I must answer, then I don't want to know", and $4=$ "I do not want to know"; we also included a "not sure" choice. The reasons for participants' preferences to receive their genetic test results were examined using multiple-choice questions. Participants' preferred method of receiving their genetic information by disease were chosen from the following: from a genetic specialist, from a family doctor, from a regional public health nurse, from a regional pharmacist, by video conference/telephone with a specialist, by mail from genetic testing companies, and on the web page of a genetic testing company. To assess genetic knowledge, we used a Japanese version of the genetic knowledge questionnaire developed by Jallinoja and Aro [12] and have been used in other researches [13, 14], consisting of 16 true or false questions regarding basic and clinical genetics.

\section{The genetics workshop}

The materials used in the genetics workshop were developed by expert consultations between a clinical geneticist, a molecular geneticist, and a certified genetic counselor at Iwate Medical University and Iwate Tohoku Medical Megabank Organization. A clinical geneticist and a molecular geneticist at Tohoku University and Tohoku Medical Megabank Organization revised the draft. The materials also referred to the announcement about revising scientific terms of genetics by The Japan Society of Human Genetics (2009) [15]. The lecture contained basic knowledge 
regarding genes, DNA, chromosomes, cells, genome, genetic diseases, genetic testing, family trees, and the necessity of carefully handling genetic information. The differences between "genetics" and "hereditary" were specifically explained in the lecture slides, because both words represent the same word, "iden" in Japanese, and therefore, people sometimes confuse the meaning of both. The lecture slides of the genetics workshop are shown as an appendix (Appendix 1-1) and an explanation of the slides is added in Appendix 1-2. The lecture slides can be also viewed at http://iwate-megabank.org/en/genetic/ [16] by clicking the "Comprehensible Explanation of Genetics" button.

The total time of the workshop was almost $60 \mathrm{~min}$. A genetics specialist presented a lecture for about $30 \mathrm{~min}$ and addressed participants' questions, and another genetic specialist and GMRCs (genome medical research coordinator) helped to manage the workshop. The participants were asked to answer the questionnaire after the lecture without referring to the distributed lecture slides. Correct answers were revealed after the participants finished answering the questionnaire.

\section{Statistical analysis}

Statistical analyses of the survey data were performed using IBM SPSS Statistics 23.0. For hypothesis testing, $p$-values $<0.05$ were considered statistically significant. Descriptive statistics were used to summarize the demographic data of the participants. Ages were sorted into six categories: (1) 20-29, (2) 30-39, (3) 40-49, (4) 50-59, (5) 60-69, and (6) $70+$. In the genetic knowledge questionnaire, correct answers received 1 point, while wrong answers and unanswered questions received 0 points. Spearman's rank-order correlation was used to assess the relationships between the genetic knowledge score and the participants' educational backgrounds, as well as their preferences to receive their genetic information by disease category. To analyze the correlation between the genetic knowledge score and the participants' educational background levels, the levels were categorized as $1=$ junior high school, $2=$ high school, $3=$ vocational college, junior college, or technical junior college, and $4=$ university, undergraduate, or graduate degree. McNemar's test and the paired $t$-test were used to investigate differences in the participants' genetic knowledge before and after the genetics workshop. A Kruskal-Wallis $\mathrm{H}$ test was used to determine the differences in participants' preferences to receive their genetic information among the six categories, and pairwise comparisons were performed using Dunn's test with a Bonferroni correction for multiple comparisons as post hoc analysis. A Wilcoxon signed-rank test was conducted to reveal the participants' preferences to receive their genetic information by disease category before and after the genetics workshop.

\section{Ethical statement}

Ethical approval was obtained from the institutional review board of Iwate Medical University School of Medicine (approval ID: H26-96). Our study was conducted in accordance with the Declaration of Helsinki, the Japanese Act on the Protection of Personal Information, and the Japan Ethical Guidelines for Medical and Health Research Involving Human Subjects. The questionnaire was accompanied by a participant information sheet on which the participants were asked to write their names and addresses, and to complete and return the questionnaire. Returning the questionnaire implied informed consent.

\section{Results}

\section{Participant characteristics}

We invited 542 people to participate in the study, and conducted a questionnaire-based assessment of the demand for genetic information with 375 participants (a response rate of $70.4 \%$ ) and their family members in the Iwate Medical University project, at the Yahaba Center and the Kesen Satellite on 28 occasions between October 2014 and February 2015. Among these respondents, 112 participated in genetics workshops, held on 10 occasions at Ofunato Hospital, Yahaba Center, and the Kesen Satellite, and reanswered the questionnaire afterward. The workshop participants' ages and educational backgrounds are shown in Tables 1 and 2. Almost $90 \%$ of our participants were over 60 years old. Of the workshop participants, $51.8 \%(n=58)$

Table 1 Age distribution of participants $(n=112)$

\begin{tabular}{lllll}
\hline Age (years) & & Male & Female & Subtotal \\
\hline $20-29$ & $N$ & 0 & 1 & 1 \\
& $(\%)$ & $(0.0)$ & $(1.4)$ & $(0.9)$ \\
$30-39$ & $N$ & 0 & 2 & 2 \\
& $(\%)$ & $(0.0)$ & $(2.9)$ & $(1.8)$ \\
$40-49$ & $N$ & 0 & 2 & 2 \\
& $(\%)$ & $(0.0)$ & $(2.9)$ & $(1.8)$ \\
$50-59$ & $N$ & 4 & 6 & 10 \\
& $(\%)$ & $(9.5)$ & $(8.6)$ & $(8.9)$ \\
$60-69$ & $N$ & 16 & 44 & 60 \\
& $(\%)$ & $(38.1)$ & $(62.9)$ & $(53.6)$ \\
Over 70 & $N$ & 22 & 15 & 37 \\
& $(\%)$ & $(52.4)$ & $(21.4)$ & $(33.0)$ \\
Total & $N$ & 42 & 70 & 112 \\
& $(\%)$ & $(37.5)$ & $(62.5)$ & $(100)$ \\
\hline
\end{tabular}


Table 2 Educational background of participants $(n=112)$

\begin{tabular}{lll}
\hline Educational background & & Subtotal \\
\hline Junior high school & $n$ & 13 \\
High school & $(\%)$ & $(11.6)$ \\
Vocational college & $n$ & 57 \\
Junior college & $(\%)$ & $(50.9)$ \\
& $n$ & 18 \\
University, undergraduate degree & $(\%)$ & $(16.1)$ \\
University, graduate degree & $n$ & 7 \\
Other (Technical junior college) & $(\%)$ & $(6.3)$ \\
& $(\%)$ & 15 \\
& $n$ & 1 \\
& $(\%)$ & $(0.9)$ \\
& $n$ & 1 \\
\end{tabular}

were recruited at Yahaba Center and 48.2\% $(n=54)$ were recruited at the Kesen Satellite.

\section{Correlation between genetic knowledge scores and educational backgrounds}

Spearman's rank-order correlation was used to assess the relationship between the participants' genetic knowledge scores and educational background levels. Before the genetics workshop, there was a positive correlation between the total genetic knowledge score and the participants' educational background levels $\left(r_{\mathrm{s}}=0.239, p=0.011\right)$. However, after the genetics workshop, there were no statistically significant correlations between the participants' total genetic knowledge scores and educational backgrounds $\left(r_{\mathrm{s}}=0.077, p=0.417\right)$.

\section{Comparison of participants' genetic knowledge before and after the genetics workshop}

We investigated the differences in the participants' genetic knowledge before and after the genetics workshop. The correct answer rates of three questions and the total genetic knowledge scores were significantly increased after the workshop, and no questions decreased in score after the workshop. The increased correct answer rates related to the statements "A gene is a disease" (before 78\%, after 93\%, $p=0.001$ ), "Different body parts include different genes" (before $34 \%$, after $77 \%, p<0.001$ ), and "It has been estimated that a person has 22,000 genes" (before $68 \%$, after $97 \%, p<0.001)$. The total genetic knowledge score increased from 11.89 to $13.30(p<0.001)$ (Table 3$)$. We also provided a comparison of genetic knowledge between our study group and those in previous studies as an appendix (Appendix 2).

\section{Participants' preferences to receive their genetic information by disease category}

The results of descriptive analysis comparing participants' preferences to receive their genetic information by disease category before and after the genetics workshop are shown in Fig. 1. In the before workshop questionnaire, the score distributions of the participants' preferences to receive their genetic information seemed dissimilar between disease categories, as assessed by visual inspection of the boxplot, and were significantly different $\left(\chi^{2}(5)=32.910\right.$, $p<0.0001)$. The results of post hoc analyses are shown in Table 4 , which revealed statistically significant differences in participants' preferences to receive their genetic information between lifestyle diseases and adult-onset nonclinically actionable diseases (303.26 vs. 354.64 ; adjusted $p=0.035$ ), lifestyle diseases and all genetic information (303.26 vs. 380.89 ; adjusted $p<0.001$ ), pharmacogenetics and adult-onset non-clinically actionable diseases (302.38 vs. 354.64 ; adjusted $p=0.029)$, pharmacogenetics and all genetic information (302.38 vs. 380.89; adjusted $p<0.001$ ), adult-onset clinically actionable diseases and all genetic information (315.51 vs. 380.89 ; adjusted $p=0.002$ ), and non-clinically actionable multifactorial diseases and all genetic information (323.58 vs. 380.89 ; adjusted $p=$ 0.014).

After the workshop, the score distributions of participants' preferences to receive their genetic information were significantly different between disease categories $\left(\chi^{2}\right.$ $(5)=52.927, p<0.001)$. Post hoc analysis revealed statistically significant differences in the participants' preferences to receive their genetic information between lifestyle diseases and adult-onset non-clinically actionable diseases (280.34 vs. 351.40 ; adjusted $p=0.01$ ), lifestyle diseases and all genetic information (280.34 vs. 497.26; adjusted $p<0.001$ ), pharmacogenetics and adult-onset non-clinically actionable diseases (280.63 vs. 351.40; adjusted $p=0.01$ ), pharmacogenetics and all genetic information ( 280.63 vs. 497.26 ; adjusted $p<0.001$ ), adultonset clinically actionable diseases and adult-onset nonclinically actionable diseases ( 288.52 vs. 351.40 ; adjusted $p=0.039)$, adult-onset clinically actionable diseases and all genetic information (288.52 vs. 497.26; adjusted $p=0.002$ ), and non-clinically actionable multifactorial diseases and all genetic information ( 331.70 vs. 497.26; adjusted $p=0.005$ ). Of the six categories, participants' preferences regarding the genetic information contained in five (lifestyle diseases, pharmacogenetics, adult-onset nonclinically actionable diseases, non-clinically actionable multifactorial diseases, and all genetic information) were 
Table 3 Comparison of participants' genetic knowledge workshop before and after the genetics

\begin{tabular}{|c|c|c|c|c|c|}
\hline & \multicolumn{2}{|c|}{$\begin{array}{l}\text { Before the } \\
\text { workshop } \\
(n=112)\end{array}$} & \multicolumn{2}{|c|}{$\begin{array}{l}\text { After the } \\
\text { workshop } \\
(n=112)\end{array}$} & \multirow[t]{2}{*}{$p$ Value $^{\mathrm{a}}$} \\
\hline & $(n)$ & $(\%)$ & $(n)$ & $(\%)$ & \\
\hline 1. One can see a gene with the naked eye & 100 & 89 & 106 & 95 & 0.210 \\
\hline 2. A gene is a disease & 87 & 78 & 104 & 93 & $0.001 * *$ \\
\hline 3. A gene is a molecule that controls hereditary characteristics & 81 & 72 & 79 & 71 & 0.860 \\
\hline 4. Genes are inside cells & 100 & 89 & 105 & 94 & 0.267 \\
\hline 5. A gene is a piece of DNA & 110 & 98 & 104 & 93 & 0.109 \\
\hline 6. A gene is a cell & 37 & 33 & 42 & 38 & 0.486 \\
\hline 7. A gene is a part of a chromosome & 101 & 90 & 103 & 92 & 0.804 \\
\hline 8. Different body parts include different genes & 38 & 34 & 86 & 77 & $<0.001 * *$ \\
\hline 9. Genes are bigger than chromosomes & 83 & 74 & 93 & 83 & 0.112 \\
\hline 10. The genotype is not susceptible to human intervention & 64 & 57 & 70 & 63 & 0.417 \\
\hline 11. It has been estimated that a person has 22,000 genes & 76 & 68 & 109 & 97 & $<0.001 * *$ \\
\hline 12. Healthy parents can have a child with a hereditary disease & 94 & 84 & 102 & 91 & 0.134 \\
\hline $\begin{array}{l}\text { 13. The onset of certain diseases is due to genes, environment, } \\
\text { and lifestyle }\end{array}$ & 94 & 84 & 102 & 91 & 0.134 \\
\hline 14. The carrier of a disease gene may be completely healthy & 98 & 88 & 105 & 94 & 0.118 \\
\hline 15. All serious diseases are hereditary & 97 & 87 & 101 & 90 & 0.503 \\
\hline $\begin{array}{l}\text { 16. The child of a disease gene carrier is always also a carrier } \\
\text { of the same disease gene }\end{array}$ & 72 & 64 & 79 & 71 & 0.381 \\
\hline Overall average score $(\%)$ & 74.3 & & 83.1 & & \\
\hline Overall average score (full score is 16 ) & 11.89 & & 13.30 & $<0.001 * * \mathrm{~b}$ & \\
\hline
\end{tabular}

Numbers refer to the percentage of participants who answered the question correctly. Before the workshop: our participants' scores before receiving a basic genetics knowledge workshop; after the workshop: our participants' scores after receiving a basic genetics knowledge workshop

${ }^{a} p$ Values for each question were calculated using McNemar's test

${ }^{\mathrm{b}}$ The overall score $p$ Values was calculated using a paired $t$-test

**: $p<0.001$

significantly decreased after the workshop compared to beforehand (Appendix 3).

\section{Participants' preferences regarding how to receive their genetic information by disease category before and after the genetics workshop}

Participants who preferred to receive their genetic results also provided information regarding how they preferred to receive their genetic information by disease category (Fig. 2). Most preferred to receive all genetic results from a genetic specialist, both before and after the genetics workshop. The numbers of participants who chose "From a genetic specialist" for lifestyle diseases, pharmacogenetics, and adult-onset clinically actionable diseases increased after the workshop compared to beforehand. For adult-onset non-clinically actionable diseases, non-clinically actionable diseases in which appearance of symptoms are not solely determined by genes, and all genetic information, the "No answer" rate increased after the workshop.

\section{Correlation between the participants' genetic knowledge scores and preferences to receive their genetic information by disease category}

There were no significant correlations between the total genetic knowledge scores and the participants' preferences to receive their genetic information by disease category.

\section{Participant characteristics}

In this study, nearly $90 \%$ of our participants were over 60 years old. This is consistent with our previous preference research based on residents of the Iwate and Miyagi prefectures, in which almost $76 \%$ of subjects were over 60 [6]. This may be because younger people are busy with work and child rearing, and could not find the time to participate in our genetics workshop. The use of a remote genetics

\section{Discussion}




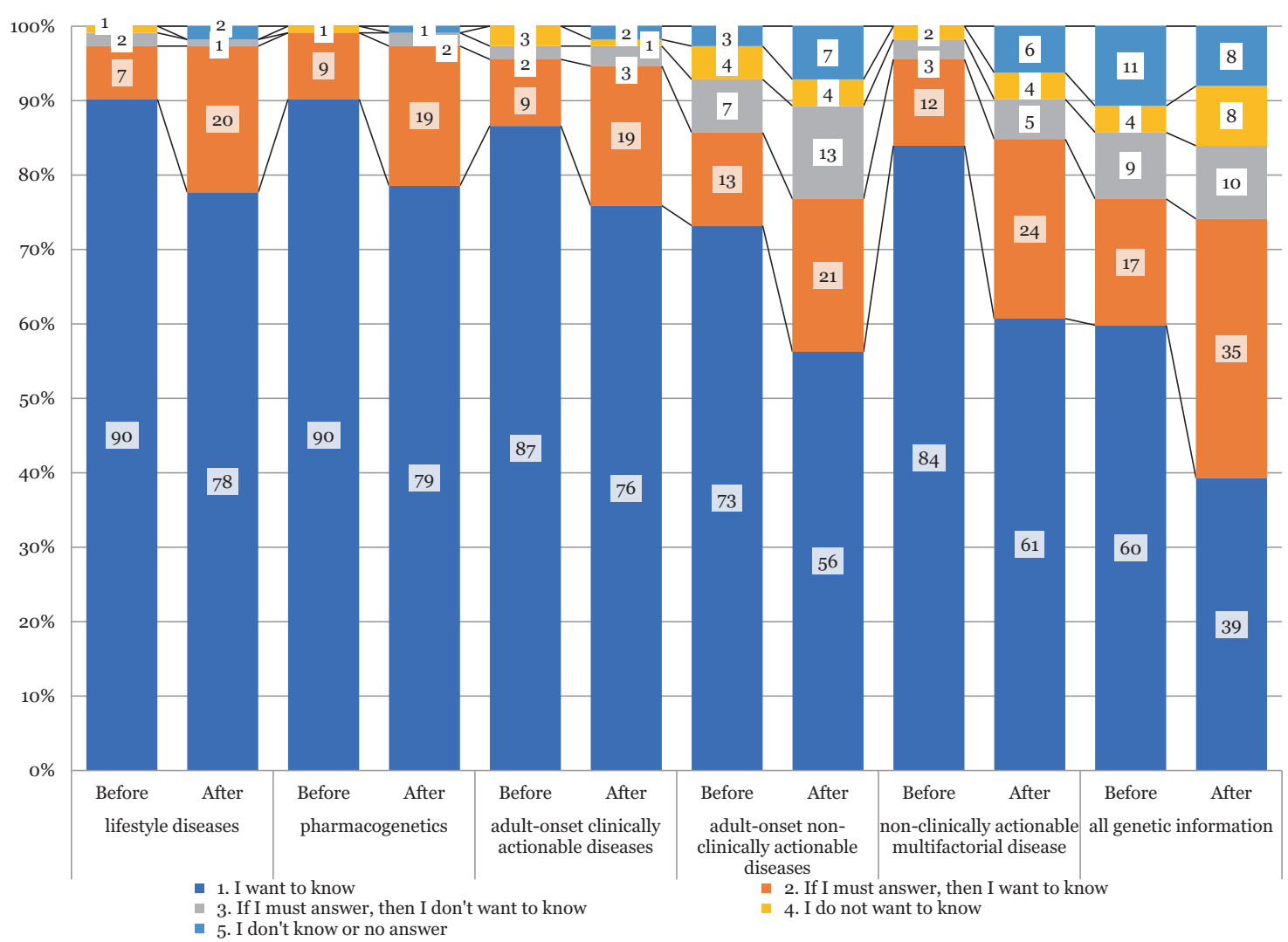

Fig. 1 Comparison among participants' preferences to receive their genetic information by disease category. Pairwise comparisons were performed using Dunn's procedure with a Bonferroni correction for multiple comparisons. Values are mean ranks unless otherwise stated

workshop using a video or website could improve the response rate of younger people.

\section{Participants' preferences to receive their genetic information by disease category before and after the genetics workshop}

Over $95 \%$ of our participants answered "I want to know" or "If I must answer, then I want to know" for lifestyle diseases, pharmacogenetics, adult-onset clinically actionable diseases, and multifactorial diseases before the workshop, and lifestyle diseases, pharmacogenetics, and adult-onset clinically actionable diseases after the workshop. The highest rate of genetic result preference was $98 \%$, both before and after the workshop. This preference rate was higher than the rates in the Tohoku Medical Megabank project's participants $(88 \%)$ and residents of the Tohoku area $(82 \%)$ in a previous study [6]. More of our participants preferred to be informed of their genetic results regarding lifestyle diseases and pharmacogenetics than adult-onset non-clinically actionable diseases and all genetic information, both before and after the genetics workshop. The availability of disease treatment has been considered a deciding factor in preference for receiving genetic results
[17]. However, in this study, treatment availability did not affect the preference to receive genetic results before the workshop. However, treatment availability may have been a deciding factor afterwards, except in the case of adult-onset clinically actionable diseases, as the preference to receive information in five of the six categories significantly decreased after the workshop. It might represent that participants perceived the information of adult-onset clinically actionable diseases as useful even after they gain additional genetic knowledge. A similar case for another disease is that for hypercholesterolemia, for which the Tohoku Medical Megabank Project has begun returning individual genetic information to the patients. Our participants also showed increased preference to receive genetic results regarding lifestyle diseases, pharmacogenetics, and adult-onset clinically actionable diseases from a genetic specialist after the workshop compared to beforehand. The "No answer" rate regarding how to receive genetic information was increased for adult-onset non-clinically actionable diseases, nonclinically actionable multifactorial diseases, and all genetic information. This tendency may represent hesitation in our participants regarding the receipt of this information after gaining accurate knowledge of human genetics at the genetics workshop. 


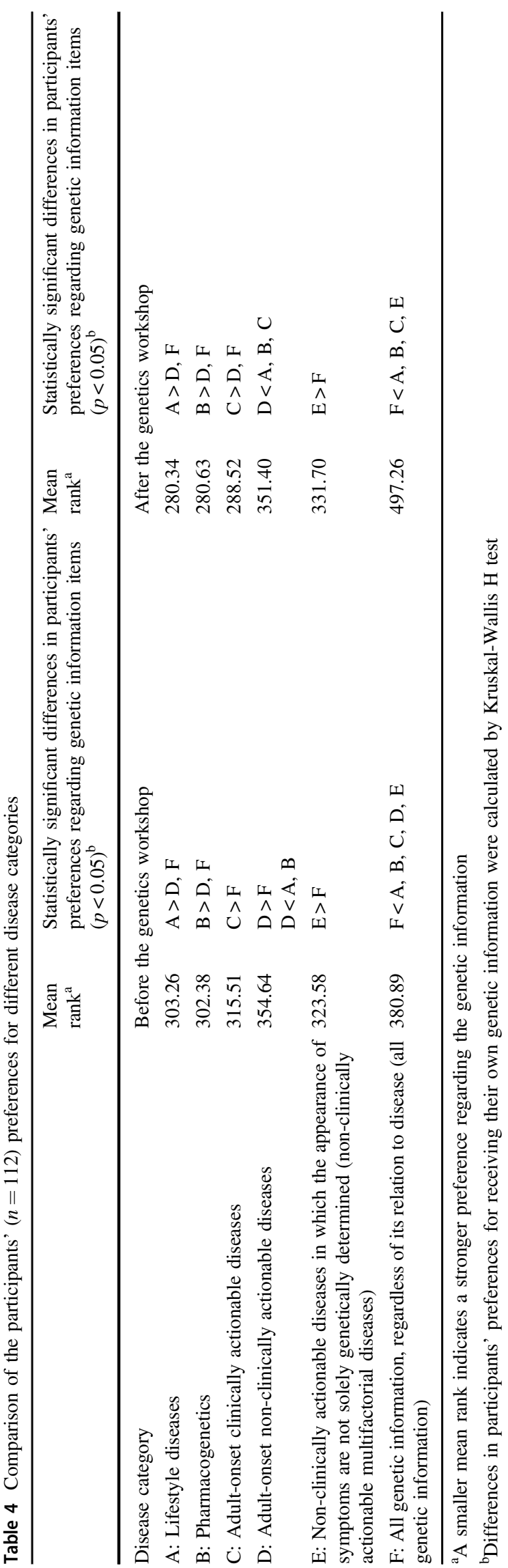

\section{Comparison of participants' genetic knowledge before and after the genetics workshop}

The total score of our participants' genetic knowledge was significantly increased despite the fact that nearly $90 \%$ of the participants were over 60 years, and it is considered that elder people find it relatively difficult to learn new knowledge. There was a positive correlation between the total genetic knowledge score and the participants' educational background level before the genetics workshop. However, after the genetics workshop, there were no significant correlations between these variables. This suggests that, regardless of their educational background, people could increase their genetic knowledge by attending the workshop. This is important, as genetic education in Japan has been non-uniformly developed, and individual differences and those between generations in genetic literacy are relatively large. There exist changes in educational curriculum among different age groups. For example, Mendelian genetics were taught in junior high school until the 1970s; however, this content disappeared from the curriculum. Mendelian genetics taught in junior high school reappeared in the textbook in 2012. The concept of the gene was also taught in the 1970s, disappeared from the curriculum, and reappeared in the late 2000s [18]. Basic knowledge of molecular genetics, inheritance, and species variation has generally increased but information on human genetics, especially disease-related concepts, is minimal in the latest government curriculum guidelines [19-21]. Harper reported that cultural isolation and extreme sensitivity over family matters, including genetic disorders, may have been delaying factors for the dissemination of medical genetics knowledge in Japan [22]. It is also known that educational backgrounds were different among the age groups. High school qualification constituted the highest percentage of educational background across all age groups in Japanese population census and among our participants. However, people in younger age groups had higher educational qualifications (Appendix 41). The second highest percentage of educational background was university undergraduate and/or graduate degrees $(18.5 \%)$ across all ages of Japanese population census (Appendix 4-2), primary and/or junior high school qualification $(28.8 \%)$ for age groups within the range of 60 to 79 years in the census (Appendix 4-3), and vocational and/or junior college qualification (22.4\%) among our participants (Table 2). Accurate knowledge of human genetics is the foundation of personalized genetic medicine. Our workshop could be an opportunity to homogenize study participants' genetic knowledge and help participants understand their genetic results. 


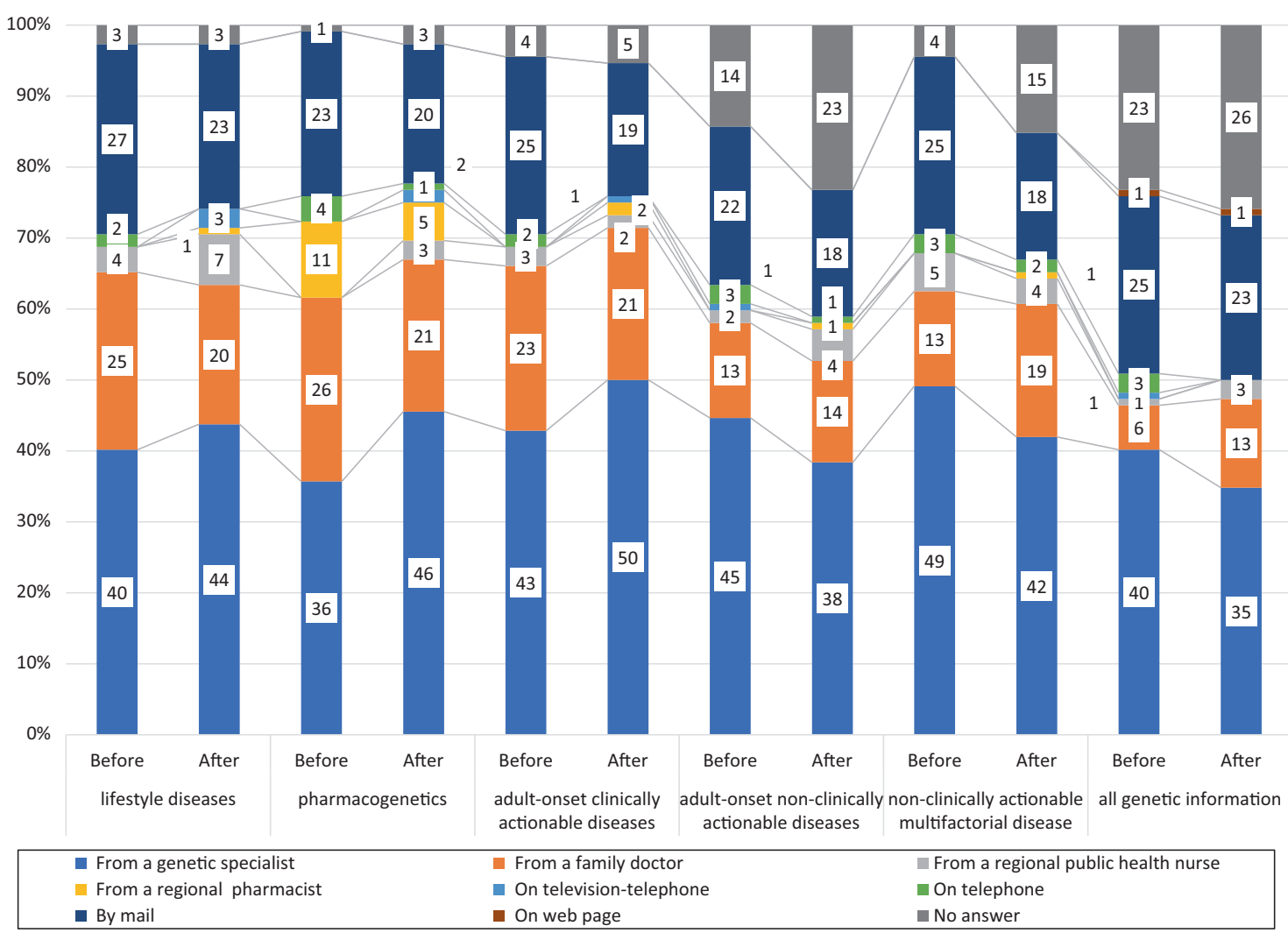

Fig. 2 The participants' preferred method of receiving their genetic information

\section{Study limitations}

There are several limitations to our study. First, our findings may have been influenced by response bias, as attending the genetics workshop demanded participant time and effort, which might have been considered inconvenient by younger and early-middle-aged people and thus created a potential disincentive for them to participate in our study. Our participants' distribution was therefore biased toward older people for whom it was relatively easy to take time to attend the workshop, and this led to increased attendance indicating that our participants may have been motivated and interested to understand their genetic information. For future research, offering a web-based genetic workshop and questionnaire could improve the response rate of those people. Second, we conducted the second questionnaire soon after the genetics workshop, and the long-term effects of the genetics workshop were not assessed. Third, all participants lived in the Tohoku region, and we did not include other areas in Japan for this study. As such, our results may not be generalizable to other regions and ages in Japan.

Acknowledgements The authors would like to thank members of Iwate Tohoku Medical Megabank Organization of Iwate Medical
University and Tohoku Medical Megabank Organization of Tohoku University for their encouragement and support. We are grateful to Prof. Fuji Nagami for his helpful discussions. We deeply thank Ms. Takako Hirano and Ms. Fumiyo Nakayama, the Genome Medical Research Coordinators of Iwate Medical University, for their help with data arrangement. We are grateful to the five senior Genome Medical Research Coordinators for their valuable suggestions, which have helped improve the quality of this research. We also thank the study participants, who played the most critical role in this research. Our work was supported in part by grants from Tohoku Medical Megabank Project (Special Account for Reconstruction from the Great East Japan Earthquake) from the Ministry of Education, Culture, Sports, Science and Technology, and the Japan Agency for Medical Research and Development. The funders had no role in study design, data collection, and analysis, decision to publish, or preparation of the manuscript. The content is solely the responsibility of the authors and does not necessarily represent the official views of the Ministry of Education, Culture, Sports, Science and Technology or the Japan Agency for Medical Research and Development.

\section{Compliance with ethical standards}

Conflict of interest The authors declare that they have no conflict of interest.

Open Access This article is licensed under a Creative Commons Attribution 4.0 International License, which permits use, sharing, adaptation, distribution and reproduction in any medium or format, as long as you give appropriate credit to the original author(s) and the source, provide a link to the Creative Commons license, and indicate if changes were made. The images or other third party material in this 
article are included in the article's Creative Commons license, unless indicated otherwise in a credit line to the material. If material is not included in the article's Creative Commons license and your intended use is not permitted by statutory regulation or exceeds the permitted use, you will need to obtain permission directly from the copyright holder. To view a copy of this license, visit http://creativecommons. org/licenses/by/4.0/.

\section{References}

1. Kalia SS, Adelman K, Bale SJ, Chung WK, Eng C, Evans JP, et al. Recommendations for reporting of secondary findings in clinical exome and genome sequencing, 2016 update (ACMG SFv2.0): a policy statement of the American College of Medical Genetics and Genomics. Genet Med. 2017;19:249-55.

2. Dewey FE, Murray MF, Overton JD, Habegger L, Leader JB, Fetterolf SN, et al. Distribution and clinical impact of functional variants in 50,726 whole-exome sequences from the DiscovEHR study. Science. 2016;354:aaf6814.

3. Sweet K, Sturm AC, Schmidlen T, McElroy J, Scheinfeldt L, Manickam K, et al. Outcomes of a randomized controlled trial of genomic counseling for patients receiving personalized and actionable complex disease reports. J Genet Couns. 2017;26:980-98.

4. Haukkala A, Kujala E, Alha P, Salomaa V, Koskinen S, Swan H, et al. The return of unexpected research results in a biobank study and referral to health care for heritable long QT syndrome. Public Health Genom. 2013;16:241-50.

5. Kuriyama S, Nagami F, Kawaguchi Y, Arai T, Kawaguchi Y, Osumi N, et al. The Tohoku Medical Megabank Project: design and mission. J Epidemiol. 2016;26:493-511.

6. Yamamoto K, Hachiya T, Fukushima A, Nakaya N, Okayama A, Tanno K, et al. Population-based biobank participants' preferences for receiving genetic test results. J Hum Genet. 2017;62:1037-48.

7. Kung JT, Gelbart ME. Getting a head start: the importance of personal genetics education in high schools. Yale J Biol Med. 2012;85:87-92.

8. Dougherty MJ. Closing the gap: inverting the genetics curriculum to ensure an informed public. Am J Hum Genet. 2009;85:6-12.

9. Dougherty MJ, Pleasants C, Solow L, Wong A, Zhang H. A comprehensive analysis of high school genetics standards: are states keeping pace with modern genetics? CBE Life Sci Educ. 2011;10:318-27.
10. Personal Genetics Education Project. http://www.pged.org/. Accessed on 26 January 2018.

11. Paneque M, Turchetti D, Jackson L, Lunt P, Houwink E, Skirton H. A systematic review of interventions to provide genetics education for primary care. BMC Fam Pract. 2016;17:89.

12. Jallinoja P, Aro AR. Does knowledge make a difference? The association between knowledge about genes and attitudes toward gene tests. J Health Commun. 2000;5:29-39.

13. Haga SB, Barry WT, Mills R, Ginsburg GS, Svetkey L, Sullivan J, et al. Public knowledge of and attitudes toward genetics and genetic testing. Genet Test Mol Biomark. 2013;17:327-35.

14. Calsbeek H, Morren M, Bensing J, Rijken M. Knowledge and attitudes towards genetic testing: a two year follow-up study in patients with asthma, diabetes mellitus and cardiovascular disease. J Genet Couns. 2007;16:493-504.

15. Idengakuyougo Kaitei no Oshirase (An announcement about revising scientific terms of genetics) in The Japan Society of Human Genetics HP. http://jshg.jp/wp-content/uploads/2017/08/ d5fdc84ae83d3a9a6627b7ac249e4db0.pdf. Accessed on 5 April 2018.

16. Genetics workshop of the Iwate Tohoku Medical Megabank Organization. http://iwate-megabank.org/en/genetic. Accessed on 26 January 2018.

17. Allen NL, Karlson EW, Malspeis S, Lu B, Seidman CE, Lehmann LS. Biobank participants' preferences for disclosure of genetic research results: perspectives from the OurGenes, OurHealth, OurCommunity project. Mayo Clin Proc. 2014;89:738-46.

18. Database of the government curriculum guidelines in National Institute for Educational Policy Research of Japan. https://www. nier.go.jp/guideline/. Accessed on 11 January 2018.

19. Ikeuchi T. Nihon no Cyuugaku Koukou ni okeru [Iden Kyouiku]: Genjyou to Mondai (Genetic education in Japanese junior and high scool: Present and Issues). Seibutu no Kagaku Iden (Sci Biol: Genet). 2015;69:166-70.

20. Lee SS, Yamamoto M. Iden Kyouiku ni okeru shingapo-ru to Nihonn no Hikaku (Comparison of Genetic Education among Singapore and Japan: Superior points of Singapore Education). Seibutu no Kagaku Iden (Sci Biol: Genet). 2015;69:190-7.

21. Santosh SK, Yamamoto M. Indo ni okeru Koutou Gakkou Kyouiku no Iden no Kagaku (High School Genetic science in India: Stepwise program regarding Genetic Education). Seibutu no Kagaku Iden (Sci Biol: Genet). 2015;69:198-203.

22. Harper PS. A short history of medical genetics. Oxford: Oxford University Press; 2008. p. 308. 